\title{
PRODUCTS OF COMPLETION REGULAR MEASURES
}

\author{
CONSTANTINOS GRYLLAKIS
}

(Communicated by R. Daniel Mauldin)

\begin{abstract}
Let $X=\prod_{i \in I} X_{i}$ and $Y=\prod_{j \in J} Y_{j}$, where all $X_{i}, Y_{j}$ are separable metric spaces. Let $\mu$ and $\nu$ be completion regular Radon probability measures on $X$ and $Y$ respectively. Then $\mu \times \nu$ on $X \times Y$ is completion regular.

This solves a problem of J. R. Choksi and D. H. Fremlin.
\end{abstract}

1. Introduction. A Radon measure $\mu$ on a completely regular space is completion regular iff every Borel set is measurable with respect to the completion of the Baire restriction of $\mu$. That is, to every Borel set $E$ there correspond two Baire sets $A$ and $B$ such that $A \subset E \subset B$ and $\mu(B-A)=0$.

In [1] J. R. Choksi posed the problem. Let $X=\prod_{i \in I} X_{i}$ and $Y=\prod_{j \in J} Y_{j}$ where all $X_{i}, Y_{j}$ are compact metric spaces. Let $\mu$ and $\nu$ be completion regular Radon measures on $X, Y$ with full support. When is $\mu \times \nu$ on $X \times Y$ completion regular? See also [3, p. 121, note after Theorem 4]. The following partial results are known.

(a) If both $\mu, \nu$ are product measures of probability measures with full support on $X, Y$ then the answer is yes: see Kakutani [7].

(b) If just one of $\mu$ and $\nu$ is a product measure the answer is yes: see Choksi and Fremlin [3].

Also Fremlin [6] has shown that for arbitrary compact spaces $X$ and $Y$ the answer is no.

In this paper it is proved that the answer to the above problem is always yes.

Moreover, the assumption that $\mu$ and $\nu$ have full support is unnecessary and the assumption of compactness of $X_{i}, Y_{j}$ is replaced by the more general assumption of separability.

For more information concerning the above problem as well as completion regular measures on product spaces the reader can consult [2].

2. In this section we will give a characterization of completion regularity of measures on uncountable products of separable metric spaces.

Let $\left\langle X_{i}\right\rangle_{i \in I}$ be a family of separable metric spaces and $X=\prod_{i \in I} X_{i}$. For every $i \in I$, let $T_{i}$ be a countable base for the topology of $X_{i}$.

For $J \subset I, \mathrm{pr}_{j}: X \rightarrow \prod_{i \in J} X_{i}$ denotes the canonical projection. If $A \subset X$ has the form $A=\operatorname{pr}_{J}^{-1}(B)$ where $B \subset \prod_{i \in J} X_{i}$ and $J \subset I$, we say that $A$ depends on (the set of coordinates) $J$.

A subset $U$ of $X$ is called elementary open (resp. basic elementary open) if it has the form $U=\operatorname{pr}_{k}^{-1}\left(\prod_{i \in k} V_{i}\right)$, where $K \subset I$ is finite and $V_{i} \subset X_{i}$ is open (resp. $\left.V_{i} \in T_{i}\right)$.

Received by the editors August 20, 1986 and, in revised form, May 4, 1987.

1980 Mathematics Subject Classification (1985 Revision). Primary 28C15. 
The set of basic elementary open sets constitute a base for the topology of $X$.

PROPOSITION. Let $\left\langle X_{i}\right\rangle_{i \in I}$ be an uncountable family of separable metric spaces and $\mu$ Radon probability measure on $X=\prod_{i \in I} X_{i}$. Then the following are equivalent

(a) The measure $\mu$ is completion regular,

(b) for every uncountable family $\left\langle U_{\alpha}\right\rangle_{\alpha \in \Lambda}$ of elementary open sets, if there is a pairwise disjoint family $\left\langle I_{\alpha}\right\rangle_{\alpha \in \Lambda}$ of finite sets of coordinates such that $U_{\alpha}$ depends on $I_{\alpha}$ then $\mu\left(\bigcup_{\alpha \in \Lambda} U_{\alpha}\right)=1$, and

(c) for every open set $U \subset X$, there exist an $U^{\prime} \supset U$ with $\mu\left(U^{\prime}\right)=\mu(U)$ and $U^{\prime}$ is a countable union of elementary open sets.

PROOF. (a) $\Rightarrow$ (b) Let $\left\langle U_{\alpha}\right\rangle_{\alpha \in \Lambda}$ be as in (b). Since $\mu$ is completion regular, it suffices to show that if $B$ is a Baire set and $B \supset\left(\bigcup_{\alpha \in \Lambda} U_{\alpha}\right)$ then $B$ must be the whole space $X$.

Indeed $B$, as a Baire set, depends on a countable set $I^{*} \subset I$ (see [8]). Thus we can find $\gamma \in \Lambda$ with $I^{*} \cap I_{\gamma}=\varnothing$ and since $B \supset U_{\gamma}$, it follows that $B \supset U_{\gamma}^{c}$ so $B=X$.

(b) $\Rightarrow$ (c) Let $U$ be an open subset of $X$. Then $U=\bigcup_{\alpha \in A} U_{\alpha}$ where $U_{\alpha} \neq U_{\beta}$ for $\alpha \neq \beta$ and each $U_{\alpha}$ is a basic elementary open set depending on a finite set $I_{\alpha} \subset I$. Without loss of generality, we assume that for some $k \in N,\left|I_{\alpha}\right|=k$ for every $\alpha \in A$. (If we set $V_{k}=\left\{\alpha \in A:\left|I_{\alpha}\right|=k\right\}$, then $U=\bigcup_{k=1}^{\infty} V_{k}$.)

Claim. For every uncountable $B \subset A$ there is an uncountable $B^{\prime} \subseteq B$ and a basic elementary open set $V$ such that $V$ depends on less than $k$ coordinates, $V \supset \bigcup_{\alpha \in B^{\prime}} U_{\alpha}$ and $\mu(V)=\mu\left(\bigcup_{\alpha \in B^{\prime}} U_{\alpha}\right)$.

First we observe that if $J$ is a finite subset of $I$, then the family of all basic elementary open sets depending on $J$ is countable. In particular, for every $\alpha \in A$, $\left\{\beta \in A: I_{\beta}=I_{\alpha}\right\}$ is countable, so there exists an uncountable $B_{1} \subseteq B$ such that $I_{\alpha} \neq I_{\beta}$ for every $\alpha, \beta \in B_{1}, \alpha \neq \beta$.

Thus by the Erdös-Rado theorem [4, p. 62] there is an uncountable $B_{0} \subseteq B_{1}$ and $J \subset I$ such that

$$
I_{\alpha} \cap I_{\beta}=J \quad \text { for every } \alpha, \beta \in B_{0} \text {. }
$$

Clearly $|J|<k$.

If $J \neq \varnothing$ then by (b) $\mu\left(\bigcup_{\alpha \in A} U_{\alpha}\right)=1$ and the claim is true for $V=X$ and $B^{\prime}=B$. (This is the case at least when $k=1$.) Now assume that $J \neq \varnothing$ and choose an uncountable $B^{\prime} \subset B_{0}$ such that

$$
\operatorname{pr}_{J}\left(U_{\alpha}\right)=\operatorname{pr}_{J}\left(U_{\beta}\right)=W \quad \text { for every } \alpha, \beta \in B^{\prime} .
$$

Set $V=\operatorname{pr}_{J}^{-1}(W)$. Clearly $V \supset \bigcup_{\alpha \in B^{\prime}} U_{\alpha}$.

For every $\alpha \in B^{\prime}$ there is an elementary open set $W_{\alpha}$ such that $W_{\alpha}$ depends on $I_{\alpha} \backslash J$ and $U_{\alpha}=V \cap W_{\alpha}$. Since $I_{\alpha} \backslash J, \alpha \in B^{\prime}$ are pairwise disjoint, (b) implies that $\mu\left(\bigcup_{\alpha \in B^{\prime}} W_{\alpha}\right)=1$. Thus

$$
\mu\left(\bigcup_{\alpha \in B^{\prime}} U_{\alpha}\right)=\mu\left(V \cap \bigcup_{\alpha \in B^{\prime}} W_{\alpha}\right)=\mu(V)
$$

and the proof of the claim is complete. 
Using the above claim, it is easily seen (using an exhaustion argument on $A$ ) that a decreasing family $\left\langle B_{\gamma}\right\rangle_{\gamma<\tau_{1}}$ of subsets of $A$ can be constructed by induction on the ordinal $\gamma$ with the following properties.

(i) $B_{0}=A$.

(ii) For every $\gamma<\tau_{1}$ there is a basic elementary open set $V_{\gamma}^{1}$ depending on less than $k$ coordinates such that

$$
V_{\gamma}^{1} \supset \bigcup_{\alpha \in B_{\gamma} \backslash B_{\gamma+1}} U_{\alpha} \text { and } \mu\left(V_{\gamma}^{1}\right)=\mu\left(\bigcup_{\alpha \in B_{\gamma} \backslash B_{\gamma+1}} U_{\alpha}\right)
$$

(iii) $B_{\gamma}=\bigcap_{\beta<\gamma} B_{\beta}$ if $\gamma$ is limit.

(iv) $C_{1} \equiv \bigcap_{\gamma<\tau_{1}} B_{\gamma}$ is countable.

Thus $\bigcup_{\gamma<\tau_{1}} V_{\gamma}^{1} \cup\left(\bigcup_{\alpha \in C_{1}} U_{\alpha}\right) \supset \bigcup_{\alpha \in A} U_{\alpha}=U$ and by the regularity of $\mu$,

$$
\mu\left(\bigcup_{\gamma<\tau_{1}} V_{\gamma}^{1} \cup\left(\bigcup_{\alpha \in C_{1}} U_{\alpha}\right)\right)=\mu(U) .
$$

If $\tau_{1}$ is a countable ordinal then (c) follows. Otherwise we repeat the same argument for the family $\left\langle V_{\gamma}^{1}\right\rangle_{\gamma<\tau_{1}}$ in the place of $\left\langle U_{\alpha}\right\rangle_{\alpha \in A}$.

Thus we find a family $\left\langle V_{\gamma}^{2}\right\rangle_{\gamma<\tau_{2}}$ of basic elementary open sets depending on less than $k-1$ coordinates and a countable $C_{2} \subset \tau_{1}$ such that

$$
\bigcup_{\gamma<\tau_{2}} V_{\gamma}^{2} \cup\left(\bigcup_{\gamma \in C_{2}} V_{\gamma}^{1}\right) \supset \bigcup_{\gamma<\tau_{1}} V_{\gamma}^{1} \text { and } \mu\left(\bigcup_{\gamma<\tau_{2}} V_{\gamma}^{2} \cup\left(\bigcup_{\gamma \in C_{2}} V_{\gamma}^{1}\right)\right)=\mu\left(\bigcup_{\gamma<\tau_{1}} V_{\gamma}^{1}\right) \text {. }
$$

Again if $\tau_{2}$ is countable (c) follows.

In this way, we may (if necessary) proceed at the $k$ th step. But then $V_{\gamma}^{k}=X$ for all $\gamma<\tau_{k}$ and (c) follows.

(c) $\Rightarrow$ (a) obvious from the regularity of $\mu$.

3. In this section we will prove the main result of this paper which is the following.

THEOREM. Let $\left\langle X_{i}\right\rangle_{i \in I}$ and $\left\langle Y_{j}\right\rangle_{j \in J}$ be families of separable metric spaces and $\mu, \nu$ completion regular Radon probabilities measures on $X=\prod_{i \in I} X_{i}$ and $Y=$ $\prod_{j \in J} Y_{j}$, respectively.

Then the product Radon measure $\mu \times \nu$ is completion regular.

For the proof of the above theorem we need a lemma.

LEMMA. Let $(X, \mu)$ and $(Y, \nu)$ be probability measure spaces and $Q \subset X \times Y$ a countable union of measurable rectangles, i.e. $Q \subset \bigcup_{m=1}^{\infty} U_{m} \times V_{m}$ where each $U_{m} \subset X$ and $V_{m} \subset Y$ is measurable, with $\mu \times \nu Q<1$.

Then there exist a sequence $\left\langle B_{n} \times A\right\rangle_{n \in N}$ of measurable rectangles in $X \times Y$ with $\mu\left(B_{n}\right) \cdot \nu(A)>0$, such that for every sequence of measurable rectangles $\left\langle B_{n}^{\prime} \times A^{\prime}\right\rangle_{n \in N}$ with $\mu\left(B_{n}^{\prime}\right) \nu\left(A^{\prime}\right)>0, B_{n}^{\prime} \subset B_{n}$ and $A^{\prime} \subset A, \mu \times \nu\left(\left(B_{n}^{\prime} \times A^{\prime}\right) \backslash Q\right)>0$ except for finitely many $n$.

Proof. For every $C \subset X \times Y$, let $C_{x}$ denote the section of $C$ at $x$, i.e. $C_{x}=$ $\{y \in Y:(x, y) \in C\}$. We set $\varepsilon=\mu \times \nu Q$ and consider a sequence $\left\langle\theta_{n}\right\rangle_{n \in N}$ of 
positive numbers such that $\theta_{1}>\varepsilon$ and $\sum_{n=1}^{\infty} \theta_{n}<1$. By induction on $n$, we will construct two decreasing sequences $\left\langle B_{n}\right\rangle_{n \in N}$ and $\left\langle S_{n}\right\rangle_{n \in N}$ of measurable sets in $X$ and $Y$, respectively, such that for every $n$

(i) $\mu B_{n}>0$,

(ii) $\nu S_{n}>1-\sum_{m=1}^{n-1} \theta_{m}$ if $n \geq 2$ and $\nu S_{1}=1$,

(iii) $\nu\left(\left(B_{n} \times S_{n}\right) \cap Q\right)_{x}\left[=\nu\left(S_{n} \cap Q_{x}\right)\right]<\theta_{n}$

for every $x \in B_{n}$.

For $n=1$, take $S_{1}=Y$ and $B_{1}=\left\{x \in X: \nu\left(Q_{x}\right)<\theta_{1}\right\}$. Since $\theta_{1}>\varepsilon$, applying Fubini's Theorem for $Q$ we have $\mu B_{1}>0$.

We now suppose that $\left\langle B_{m}\right\rangle_{m<n}$ and $\left\langle S_{m}\right\rangle_{m<n}$ where $n \geq 2$ been constructed.

We set

$$
P_{k}=\left(B_{n-1} \times S_{n-1}\right) \cap\left(Q \backslash \bigcup_{m=1}^{k}\left(U_{m} \times V_{m}\right)\right)
$$

and observe that $\lim _{k \rightarrow \infty} \mu \times \nu P_{k}=0$.

Fix a $k$ such that

$$
\mu \times \nu P_{k}<\theta_{n} \cdot \mu B_{n-1} .
$$

For every $F \subset\{1, \ldots, k\}$ set

$$
\begin{aligned}
& R_{F}=\left\{x \in B_{n-1}: x \in U_{m} \Leftrightarrow m \in F \forall m \in\{1, \ldots, k\}\right\} \\
& \left.\qquad R_{F}=\left(\bigcap_{m \in F}\left(U_{m} \cap B_{n-1}\right)\right) \backslash\left(\bigcup_{\substack{1 \leq m \leq k_{n} \\
m \notin F}}\left(U_{m} \cap B_{n-1}\right)\right]\right] \\
& T_{F}=S_{n-1} \cap\left(\bigcup_{m \in F} V_{m}\right) \text { and } \\
& J_{F}=\left(R_{F} \times\left(S_{n-1} \backslash T_{F}\right)\right) \cap Q=\left(R_{F} \times\left(S_{n-1} \backslash T_{F}\right)\right) \cap\left(\bigcup_{m>k}\left(U_{m} \times V_{m}\right)\right) .
\end{aligned}
$$

We observe that the set of all $R_{F}$ is a finite measurable partition of $B_{n-1}$ and the set of all $J_{F}$ a finite measurable partition of $P_{k}$. Thus, if we assume that for every $F \mu \times \nu J_{F} \geq \theta_{n} \cdot \mu R_{F}$, then summing over $F$ we conclude that $\mu \times \nu P_{k} \geq \theta_{n} \cdot \mu B_{n-1}$, contradicting (*). Therefore there exist some $F$ such that $\mu \times \nu J_{F}<\theta_{n} \cdot \mu R_{F}$. In particular, $\mu R_{F}>0$.

We set $S_{n}=S_{n-1} \backslash T_{F}$.

Since $\mu \times \nu\left(\left(R_{F} \times S_{n}\right) \cap Q\right)<\theta_{n} \cdot \mu R_{F}$, using Fubini's Theorem we find a measurable set $B_{n} \subset R_{F}$ such that $\mu\left(B_{n}\right)>0$ and $\mu\left(\left(B_{n} \times S_{n}\right) \cap Q\right)_{x}<\theta_{n}$ for every $x \in B_{n}$.

Let $x \in B_{n}$. Since $B_{n} \subset R_{F} \subset B_{n-1}$ we have $T_{F} \subset S_{n-1} \cap Q_{x}$ and, by the induction hypothesis

$$
\nu\left(T_{F}\right) \leq \nu\left(S_{n-1} \cap Q_{x}\right)<\theta_{n-1} \quad \text { and } \quad \nu\left(S_{n}\right)=\nu\left(S_{n-1}\right)-\nu\left(T_{F}\right) \geq 1-\sum_{m=1}^{n-1} \theta_{m} .
$$

The construction of $\left\langle B_{n}\right\rangle_{n \in N}$ and $\left\langle S_{n}\right\rangle_{n \in N}$ is now complete. 
We set $A=\bigcap_{n=1}^{\infty} S_{n}$ and prove that $\left\langle B_{n} \times A\right\rangle_{n \in N}$ satisfies the conclusion of the lemma. By (ii) $\nu(A)=1-\sum_{m=1}^{\infty} \theta_{m}>0$.

Let $\left\langle B_{n}^{\prime} \times A^{\prime}\right\rangle_{n \in N}$ be a sequence of measurable rectangles such that $\mu\left(B_{n}^{\prime}\right) \nu\left(A^{\prime}\right)>$ $0, B_{n}^{\prime} \subset B_{n}$ and $A^{\prime} \subset A$. By (iii) for every $n$ we have

$$
\nu\left(\left(B_{n}^{\prime} \times A^{\prime}\right) \cap Q\right)_{x}<\theta_{n} \quad \text { for every } x \in B_{n}^{\prime},
$$

so by Fubini's Theorem,

$$
\mu \times \nu\left(\left(B_{n}^{\prime} \times A^{\prime}\right) \cap Q\right)<\theta_{n} \cdot \mu\left(B_{n}^{\prime}\right) .
$$

If $n$ is such that $\mu\left(B_{n}^{\prime} \times A^{\prime} \backslash Q\right)=0$, then

$$
\mu\left(B_{n}^{\prime}\right) \nu\left(A^{\prime}\right)=\mu \times \nu\left(B_{n}^{\prime} \times A^{\prime}\right)=\mu \times \nu\left(\left(B_{n}^{\prime} \times A^{\prime}\right) \cap Q\right)<\theta_{n} \cdot \mu\left(B_{n}^{\prime}\right)
$$

so $\nu\left(A^{\prime}\right)<\theta_{n}$. But this can happen for only a finite number of $n$ 's.

Note. It is interesting to compare the above lemma with the following fact proved in [5].

THEOREM (ERDÖS-OXTOBY). Let $(X, \mu)$ and $(Y, \nu)$ be finite diffuse measure spaces and $\varepsilon>0$. Then there exist a sequence $\left\langle I_{n} \times J_{n}\right\rangle_{n \in N}$ of measurable rectangles such that

(i) $\sum_{n \in N} \mu I_{n} \nu J_{n} \leq \varepsilon$.

(ii) If $E \times F$ is a measurable rectangle with $\mu E \cdot \nu F>0$, then there is an $n \in N$ such that $\mu\left(E \cap I_{n}\right) \nu\left(F \cap J_{n}\right)>0$, i.e. $\mu \times \nu(Q \cap(E \times F))>0$ where $Q=\bigcup_{n \in N} I_{n} \times J_{n}$. (For a simple proof due to $R$. O. Davis see [6].)

The preceding lemma says that we cannot have an extension of Erdös-Oxtoby theorem in a certain sense.

We now come to the proof of the main theorem.

ProOF. We suppose that both $I$ and $J$ are uncountable since if one of $I, J$ is countable it is quite easy to prove our theorem. It will be enough to prove that $(X \times Y, \mu \times \nu)$ satisfies (b) of proposition of $\S 2$.

We suppose if possible otherwise. Then there exists a family $\left\langle W_{\alpha}\right\rangle_{\alpha<\omega_{1}}$ of elementary open sets in $X \times Y$ such that

(i) $W_{\alpha}=U_{\alpha} \times V_{\alpha}$, where $U_{\alpha}, V_{\alpha}$ are elementary open sets in $X$ and $Y$, respectively, $U_{\alpha}$ depends on a finite set $I_{\alpha} \subset I, V_{\alpha}$ on a finite set $J_{\alpha} \subset J$ and for every $\alpha \neq \beta\left(I_{\alpha} \cup J_{\alpha}\right) \cap\left(I_{\beta} \cup J_{\beta}\right)=\varnothing$ and

(ii) $\mu \times \nu\left(\bigcup_{\alpha<\omega_{1}} W_{\alpha}\right)<1$.

Because $\mu \times \nu$ is Radon there exists a countable $M \subset \omega_{1}$ such that $\mu \times \nu\left(\bigcup_{\alpha<\omega_{1}} W_{\alpha}\right)$ $=\mu \times \nu\left(\bigcup_{\alpha \in M} W_{\alpha}\right)$.

Let $\left\langle B_{n} \times A\right\rangle_{n \in N}$ be a sequence of measurable rectangles in $X \times Y$ as in the statement of the lemma for $Q=\bigcup_{\alpha \in M} W_{\alpha}$.

Claim 1. There exists an $\alpha<\omega_{1}$ such that

$$
\nu\left(V_{\gamma} \cap A\right)>0 \text { for every } \gamma>\alpha .
$$

Indeed, supposing otherwise, there exists an uncountable $\Delta \subset \omega_{1}$ such that $\nu\left(V_{\gamma} \cap A\right)=0$ for every $\gamma \in \Delta$. Because $\nu$ is Radon there exist a countable $\Delta^{\prime} \subset \Delta$ such that $\nu\left(\bigcup_{\gamma \in \Delta} V_{\gamma}\right)=\nu\left(\bigcup_{\gamma \in \Delta^{\prime}} V_{\gamma}\right)$ but $\nu\left(V_{\gamma} \cap A\right)=0 \forall \gamma \in \Delta^{\prime}$ so $\nu\left(\bigcup_{\gamma \in \Delta} V_{\gamma}\right)=\nu\left(\bigcup_{\gamma \in \Delta}, V_{\gamma}\right) \leq 1-\nu A<1$ which contradicts proposition of $\S 2$ because $\nu$ is completion regular. 
For similar reasons the following claim is valid.

ClaIM 2. For every $n \in N$ there exists a $\beta_{n}<\omega_{1}$ such that $\mu\left(U_{\gamma} \cap B_{n}\right)>0$ for every $\gamma>\beta_{n}$.

We choose a $\delta>\sup \left\{\alpha, \beta_{n}, n \in N\right\}, \delta<\omega$, and set $A^{\prime}=: A \cap U_{\delta}$ and $B_{n}^{\prime}=$ $B_{n} \cap V_{\delta}$ for every $n \in N$. Then $\mu\left(B_{n}^{\prime}\right) \cdot \nu\left(A^{\prime}\right)>0, B_{n}^{\prime} \times A^{\prime} \subset B_{n} \times A$ and $B_{n}^{\prime} \times A^{\prime} \subset U_{\delta} \times V_{\delta} \subset \bigcup_{\alpha<\omega_{1}} W_{\alpha}$ for every $n$ so $\mu \times \nu\left(B_{n}^{\prime} \times A^{\prime} \backslash Q\right)=0$ for every $n$. But this is impossible by the choice of $\left\langle B_{n} \times A\right\rangle_{n \in N}$. This ends the proof of our theorem.

ACKnowledgment. The author wishes to thank Professor D. H. Fremlin for very useful correspondence concerning the subject of this paper and especially Professor G. Koumoullis for considerable help in writing it. Also the author wishes to thank the referee for finding a gap in an earlier proof of proposition of $\S 2$.

\section{REFERENCES}

1. J. R. Choksi, Problem 1 in "Problem Section", Measure theory and its applications (J. M. Belley, J. Dubois and P. Morales, eds.), Lecture Notes in Math., vol. 1033, Springer-Verlag, Berlin and New York.

2. L_ Recent developments arising out of Kakutani work on completion regularity of measure, Contemp. Math., vol. 26, Amer. Math. Soc., Providence, R.I., 1984, pp. 81-94.

3. J. R. Choksi and D. H. Fremlin, Completion regular measures on product spaces, Math. Ann. 241 (1979), 113-128.

4. W. W. Comfort and S. Negrepontis, The theory of ultrafilters, Springer-Verlag, Berlin and New York, 1984.

5. P. Erdös and J. C. Oxtoby, Partitions of the plane into sets having positive measure in every non-null measurable product set, Trans. Amer. Math. Soc. 79 (1955), 91-102.

6. D. H. Fremlin, Products of Radon measures: a counter-example, Canad. Math. Bull 19 (1976), 285-289.

7. S. Kakutani, Notes on infinite product measures. II, Proc. Imperial Acad. Tokyo 19 (1943), 184-188.

8. K. A. Ross and A. H. Stone, Products of separable spaces, Amer. Math. Monthly 71 (1964), 398-403.

Box 64075, Zografou 15710, Athens, Greece 